\title{
Epiphora; Successful Response to Systemic Steroids vs Topical Steroids
}

\section{Ali Arif ${ }^{*}$, Humara Gull ${ }^{2}$}

${ }^{1}$ Northeast Ohio Medical University, Ohio, United States

${ }^{2}$ Primary Care Physician, iMD Healthcare and Imaging, United States

Correspondence to: Ali Arif, Northeast Ohio Medical University, Ohio, United States.

Received date: August 26, 2021; Accepted date: September 6, 2021; Published date: September 13, 2021

Citation: Arif A, Gull H (2021) Epiphora; Successful Response to Systemic Steroids vs Topical Steroids. J Med Res Surg 2(5): pp. 1-3. doi: 10.52916/jmrs214056

Copyright: (C2021 Arif A, et al. This is an open-access article distributed under the terms of the Creative Commons Attribution License, which permits unrestricted use, distribution and reproduction in any medium, provided the original author and source are credited.

\section{ABSTRACT}

Purpose: Topical steroids are considered to be the first line of treatment for some forms of idiopathic punctal stenosis causing epiphora. We present a case of epiphora, which was unresponsive to traditional management, but responded to one week of systemic steroids.

Observations: A 48-year-old male with no significant medical history, was referred to us for excessive tearing. He was seen earlier by an ophthalmologist for redness in one of his eyes. He was prescribed Pataday (Olopatadine) eye drops and Zylet (Tobramycin, Lotepriol) eye drops for both eyes. The patient was seen again after a week of treatment without much improvement. His treatment was changed to Alrex (Loteprednol) and Azasite (Azithromycin) eye drops. The redness of the eye disappeared in a week but patient noticed excessive tearing of his eyes. He was referred to reconstructive ophthalmologist for treatment of possible punctal stenosis. On examination the height of tear meniscus was increased and diagnostic lacrimal probing and syringing of the lacrimal drainage system demonstrated both lacrimal drainage system patent. Punctal stenosis was diagnosed. Over the next couple of weeks, patient underwent multiple syringing of the lacrimal duct system with temporary relief of symptoms. He was also prescribed Tobradex (Tobramycin+Dexamethasone) eye drops to be used for a month. Eye drops helped for the duration of treatment but symptoms returned after a couple of days of discontinuing eye drops. Patient also received ductal probing and punctal dilation in the office without much relief. Mini perforated plugs were placed in both puncta resulting in temporary relief. At the same time the intra ocular pressure started to rise and Tobradex eye drops were discontinued immediately. After almost three months of treatment and continuation of debilitating symptoms of epiphora, patient decided to consider surgical intervention and consulted an ENT surgeon for second opinion. Since he was responding to topical steroids, which was causing an increase in his intra ocular pressure, the ENT surgeon suggested Medrol Pack (Prednisolone for a week). Patient was symptom free after 4 days of treatment and by 7 th day, his eyes were back to normal with complete disappearance of epiphora symptoms.

Conclusions and importance: Systemic steroids can be beneficial in patients suffering from Idiopathic/latrogenic punctal stenosis unresponsive to topical steroids and conventional treatments.

\section{Keywords:}

Epiphora, Punctal stenosis, Steroids, Nasolacrimal duct.

\section{Introduction}

Severe epiphora can be a debilitating condition because it not only causes patient discomfort but also visual disturbance. The lacrimal system consists of the main lacrimal gland, the accessory lacrimal glands and the conjunctival epithelium, responsible for producing tears. Tears are spread over the surface of the eye by blinking to establish the precorneal tear film. Tears are then drained through nasolacrimal ducts, a canicular connection between conjunctiva and nasal cavity. About 90 percent of tears are reabsorbed through nasolacrimal duct mucosa and 10 percent drain into the floor of nasal cavity. Any imbalance between tear production and loss can result in epiphora. Most imbalances fall into the categories of punctal obstruction, canicular obstruction, nasolacrimal duct obstruction, poor pump function, lid malposition, and ocular surface disorders. Punctal stenosis is considered one of the most common causes of epiphora but the incidence is still unknown, ranging from $8 \%$ to $54.3 \%$ [1]. Old age is identified as the most common cause of punctal stenosis with a mean age at diagnosis of 69.4 [2]. Multiple causes of punctal stenosis and canicular obstruction have been reported including, inflammation, infection, topical and systemic medications, neoplastic conditions, systemic diseases, trauma, and idiopathic [1].

\section{Case Report}

A 48-year-old male with no significant past medical history, was seen by his ophthalmologist for redness in one of his eyes, who prescribed Pataday (Olopatadine) eye drops and Zylet (Tobramycin, Lotepriol) eye drops for both eyes. The patient returned after a week of treatment without much improvement. His treatment was changed to Alrex (Loteprednol) and Azasite (Azithromycin) eye drops. The redness of the eye disappeared in a week but patient noticed excessive tearing of his eyes. He was referred to reconstructive ophthalmologist for further treatment of possible punctal stenosis. On examination patient's VA was 
$20 / 20$ in both eyes. IOP were within normal limits in both eyes. The height of tear meniscus was increased and diagnostic lacrimal probing and syringing of the lacrimal drainage system demonstrated that both sides were patent and draining. Mild form of MGD was considered and punctal stenosis was diagnosed. Over the next couple of weeks, patient underwent multiple syringing, but the symptoms kept returning after a couple of days of treatment. He was also prescribed Tobradex eye drops to be used for a month. Eye drops helped relieve the symptoms only for the duration of treatment and the symptoms came back after a couple of days of discontinuing eye drops.

Patient also received ductal probing and punctal dilation in the office with temporary relief. Mini perforated plugs were placed in both puncta resulting again, in temporary relief. At the same time patient's intra ocular pressure started to rise with c/o sensitivity to light. Tobradex eye drops were discontinued immediately. After almost three months of treatment and continuation of debilitating symptoms of epiphora, patient decided to consider DCR and consulted an ENT surgeon for second opinion. Since he was responding to topical steroids, which in turn were causing an increase in his intra ocular pressure, the ENT surgeon prescribed Medrol Pack (Prednisolone) to be taken for a week. Patient was symptom free after 4 days of treatment and by 7 th day, his eyes were back to normal without any signs of epiphora.

\section{Discussion}

Punctal stenosis is considered one of the most common causes of epiphora but the incidence is still unknown, ranging from $8 \%$ to $54.3 \%$ [1]. Old age is identified as the most common cause of punctal stenosis with a mean age at diagnosis of 69.4 [2]. Multiple other causes of punctal stenosis have been reported including, inflammation (Ch. Blepharitis, Ocular Cicatricial Pemphigoid, Graft vs Host Disease, Dry Eye Syndrome, Eye Lid Malposition), infection (Chlamydia Trachomatis, Actinomyces, Herpes Virus, Human Papilloma Virus), topical medications (Timolol, Latanoprost, Betaxolol, Dipivefrine Hydrochloride, Echothiopate lodide, Pilocarpine, Prednisolone AcetatePhenylephrine Hydrochloride, Adrenaline, Chloramphenicol, Tobramycin, Indomethacin, Dexamethasone, Tropicamide, Naphazoline, Artificial Tears, Mitomycin-C) systemic medications (5-Fluorouracil, Docetaxel, Piclitaxel, Idoxuridine, Trastuzumab Emtansine) neoplastic (Peripunctal Tumors) systemic diseases (Acrodermatitis Enteropathica, Porphyria Cutanea Tarda) other (Local Irradiation, Photodynamic Therapy for Macular Disease) trauma, and idiopathic [1]. The etiology in this case appears to be the reaction to one of the anti-allergy drops or topical antibiotics. As mentioned earlier, many medications including artificial tears can lead to this condition. This reaction was causing inflammation of the epithelial lining of the nasolacrimal drainage system (Figure 1), leading to obstruction. There were no signs of infection or any obstructive etiology

Instillation of topical steroid (combination of Tobramycin and Dexamethasone) was effective in providing symptomatic relief and quantitative decrease in of tear meniscus in this case. In literature, a study reported a 64\% efficacy with topical steroids in nasolacrimal drainage obstruction [3]. Inflammation and edema of lacrimal drainage are the main pathological findings in the early phase of lacrimal drainage system stenosis [4], which can be reversed by anti-inflammatory treatment such as

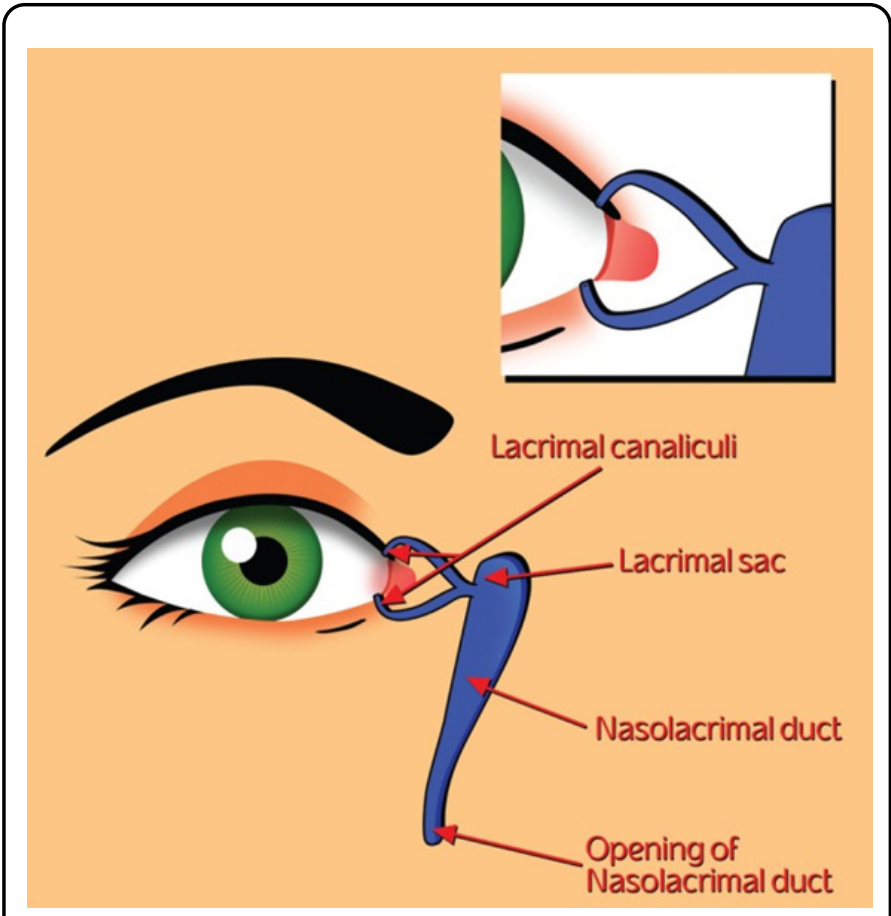

Figure 1: The puncti are positioned medially, near the medial canthus. They are located within the papillae. This complex opens into the tear layer. The tears are collected through the puncti and into the canaliculi [1].

topical steroids. In addition, perforated punctal plugs have been used for punctal stenosis with good results [5]. Other options including mini-monoka stents and punctal snip procedures have also been reported for the treatment of refractory epiphora $[6,7]$. All these options were discussed with this patient. The appearance of symptoms in this patient was immediately after the use of topical medication (anti-allergy, antibiotic). It is possible that epiphora and lacrimal drainage stenosis in this case were coincidental findings induced by other etiologies such as blepharitis. However, no other possible etiologies such as infection, endogenous or exogenous inflammation, internal or external mechanical problems or trauma was detected in patient's history or physical examination. In this case the diagnosis and subsequent treatment with topical steroids not only proved to be therapeutic but also diagnostic. Given the increase in IOP in this case and considering the long-term side effects of topical steroids including early cataract and glaucoma $[8,9]$, it was prudent to give a trial of systemic steroids. Steroids have a long record of effective anti-inflammatory agent in many conditions [10].

\section{Conclusion}

We believe that all the possible conservative management of lacrimal drainage stenosis including topical and systemic steroids (Medrol pack) should be exhausted before considering any surgical intervention.

\section{Acknowledgements}

Thank you to Dr. Humara Gull for providing the opportunity to construct a case report and to the patient for their consent.

\section{Patient Consent:}

Consent to publish this case report has been obtained from the patient in writing 


\section{Conflict of Interest:}

The authors have no financial disclosures.

\section{Funding:}

No funding or grant support.

\section{Authorship:}

All authors attest that they meet the current ICMJE criteria for Authorship.

\section{References}

1. Soiberman U, Kakizaki H, Selva D, et al. (2012) Punctal stenosis: definition, diagnosis, and treatment: Review. Clin Ophthalmol 6: pp. 1011-1018.

2. Kashkouli MB, Beigi B, Murthy R, et al. (2003) Acquired external punctal stenosis: etiology and associated findings. Am J Ophthalmol 136(6): pp. 1079-1080.

3. Dayal Y (1962) Corticosteroids and Fibrolysin in the prevention of lacrimal duct obstruction. Br J Ophthalmol 46(1): pp. 27-30.

4. Linberg JV, McCormick SA (1986) Primary acquired nasolacrimal duct obstruction. A clinicopathologic report and biopsy technique. Ophthalmol 93(8): pp. 1055-1063.

5. Konuk O, Urgancioglu B, Unal M (2008) Long-term success rate of perforated punctal plugs in the management of acquired punctal stenosis. Ophthal Plast Reconstr Surg 24(5): pp. 399402.

6. Kristan R, Branch L (1988) Treatment of lacrimal punctal stenosis with a one-snip canaliculotomy and temporal puntal plug. Arch Ophthalmol 106(7): pp. 878-879.

7. Hussain RN, Kanani H, McMullan T (2012) Use of minimonoka stents for punctal/canalicular stenosis. Br J Ophthalmol 96(5): pp. 671-673.

8. Greiner JV, Chylack Jr LT (1979) Posterior subcapsular cataracts: histopathologic study of steroid-associated cataracts Arch Ophthalmol 97(1): pp. 135-144.

9. Razeghinejad MR, Katz LJ (2012) Steroid-induced iatrogenic glaucoma. Ophthalmic Res 47(2): pp. 66-80.

10. Barnes PJ (1998) Anti-inflammatory actions of glucocorticoids: molecular mechanisms Clin Sci (Lond) 94(6): pp. 557-572. 